\title{
Uma proposta para o uso de Elementos Inteligentes em Domínios Restritos da Infraestrutura da Internet
}

\author{
Julião Braga $^{1}$, Nizam Omar ${ }^{1}$, Lisandro Zambenedetti Granville ${ }^{3}$ \\ ${ }^{1}$ Departamento de Engenharia Elétrica e Computação \\ Universidade Prebisteriana Mackenzie (UPM) São Paulo, SP - BR \\ ${ }^{2}$ Instituto de Informática - Universidade Federal do Rio Grande do Sul \\ Porto Alegre, RS - BR \\ juliao@braga.eti.br, nizam.omar@mackenzie.br, granville@inf.ufrgs.br
}

\begin{abstract}
This project aims to propose and develop a model of interconnection, interaction and cooperation between Intelligent Elements (multi-agents) able to make the functions, features and facilities of the Internet Infrastructure autonomous processes and oriented to minimize human intervention, providing the conditions that allow administrators of Autonomous System to ensure an Internet better for its users. The model provides a mechanism for multi-agent cooperation, independently of a high-level central coordination, and ensures environmental safety requirements.
\end{abstract}

Resumo. Este projeto tem como objetivo propor e desenvolver um modelo de interconexão, interação e cooperação entre os elementos inteligentes (multiagentes) caracterizando as funções e facilidades da infraestrutura de Internet, processos autónomos orientadas para minimizar a intervenção humana, proporcionando as condições que permitem administradores do Sistema Autônomo garantir uma Internet melhor para seus usuários. O modelo fornece um mecanismo para a cooperação multi-agente, independentemente de uma coordenação central de alto nível, e garante requisitos de segurança do ambiente.

\section{Introdução}

Em 2001 Paul Horn, inspirado na fisiologia de sistemas vivos apresentou uma proposta para o futuro dos produtos da IBM, da qual ele era vice-presidente. Na proposta, os esforços de especialistas na manutenção, controle e operação de sistemas de computação poderiam ser minimizados e consequentemente ter seus custos reduzidos. Horn denominou esta solução de Computação Autonômica [Horn 2001]. As contribuições expandiram-se aglomerando pesquisas multidisciplinares e os resultados mostraram-se surpreendentes [Movahedi et al. 2012], a ponto de aplicar tais ideias na solução de diversos problemas da computação (principalmente software), e viabilizaram, por exemplo, tecnologias, como a das sondas espaciais [Sterritt et al. 2005], particularmente, os veículos espaciais não tripulados (Unmanned Space Vehicles - USVs) [Insaurralde and Vassev 2015].

O interesse em Computação Autonômica tem conduzido a aplicação de Agentes Inteligentes na Infraestrutura da Internet concentram-se em protocolos e, 
neste sentido, técnicas como Software Defined Networking (SDN) [Shukla 2014] [Nadeau and Gray 2013] [Wickboldt et al. 2015] impulsionarão o desenvolvimento de iniciativas nesta direção.

Este artigo tem como objetivo propor uma arquitetura com funções de interconexão, interação e cooperação entre Agentes Inteligentes com o compromisso de minimizarem a intervenção humana sobre os recursos e facilidades, no âmbito do domínio restrito aos Sistemas Autônomos (ASes), que constituem a Internet, uma rede de redes. Como se adicionam outras características aos agentes inteligentes deu-se denominação genérica de Elementos Inteligentes (Intelligent Elements - IEs). Os IEs são orientados em minimizar a intervenção humana e a disponibilizar condições para que os administradores de ASes contribuam de forma significativa na direção de tornar a Internet, cada vez melhor. O modelo assegura um mecanismo de cooperação entre múltiplos IEs de forma independente, distinguindo-se das propostas, onde agentes inteligentes aderem e são dependentes de uma coordenação central, à qual se agrega intervenção humana. À solução proposta dá-se o nome de Arquitetura de Redes Autônomas sobre Domínios Restritos (Autonomous Network Architecture over Restricted Domains - ANARD).

O desenvolvimento do projeto avança em considerações mais profundas propondo a continuação do desenvolvimento do modelo que envolverá, necessariamente, estudos e pesquisas de médio/longo prazos.

As próximas seções deste artigo estarão organizadas conforme a seguir. Na seção 2 serão contextualizados o domínio de aplicação e suas características principais. Na seção 3 tem-se o referencial teórico com as definições fundamentais dos conceitos e técnicas envolvidos. A seção 4 inclui uma revisão dos trabalhos relacionados. A seção 5 descreve o modelo ANARD sob o aspecto abstrato e de implementação. A seção 6 são as conclusões do trabalho. A seção 7 é uma abordagem sobre os futuros desafios.

\section{O domínio da aplicação e suas características}

\subsection{A arquitetura da Internet}

Segundo o Internet Engineering Task Force (IETF), a definição de Internet está associada à noção de Sistema Autônomo (AS, do inglês Autonomous System). A definição sucinta de um AS, encontrada em [Hawkinson and Bates 1996] é, em tradução livre:

Um AS é um grupo de um ou mais prefixos IP, administrado por um ou mais operadores de rede, o qual tem uma única política de roteamento, claramente definida. ${ }^{1}$

$\mathrm{Na}$ definição acima, IP (Internet Protocol) é um protocolo, isto é, um conjunto de especificações e programas usados na Internet. O termo prefixo IP é usado para designar um conjunto (ou bloco) de números IPs (identificação numérica necessária para que um dispositivo ou programa seja reconhecido na Internet).

A Figura 1 exibe a topologia da Internet com base nesta definição de AS.

\footnotetext{
${ }^{1}$ Original: An AS is a connected group of one or more IP prefixes run by one or more network operators which has a single and clearly defined routing policy
} 


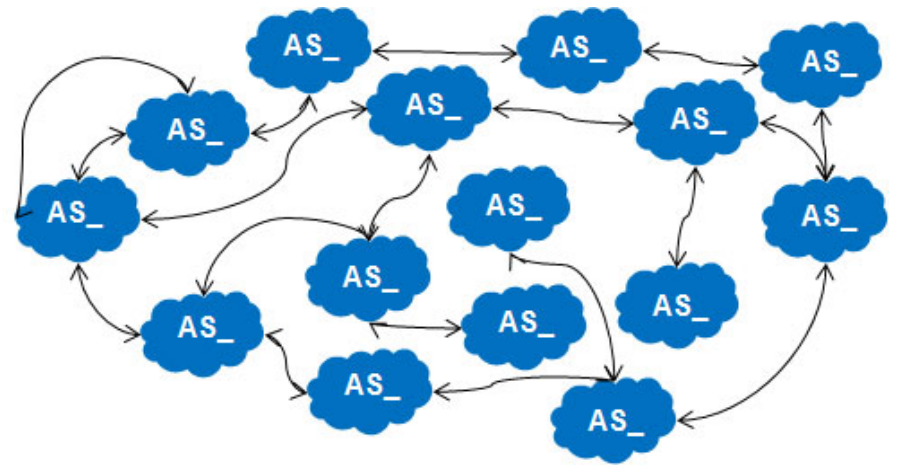

Figura 1. A Internet é uma rede de Sistemas Autônomos. O símbolo representa um número $i$, onde $\forall i \mid 0 \leq i<4.294 .967 .296 \equiv 2^{32}$. Assim, diz-se que o $i$ é o número do AS ou, mais comumente, $i$ é um ASN (Autonomous System Number)

\subsubsection{Como os ASes se interconectam}

Da Figura 1 obtém-se a Figura 2 que exibe um exemplo de interconexão entre dois ASes (ASx e ASy, onde $x \neq y$ ), com mais detalhes.

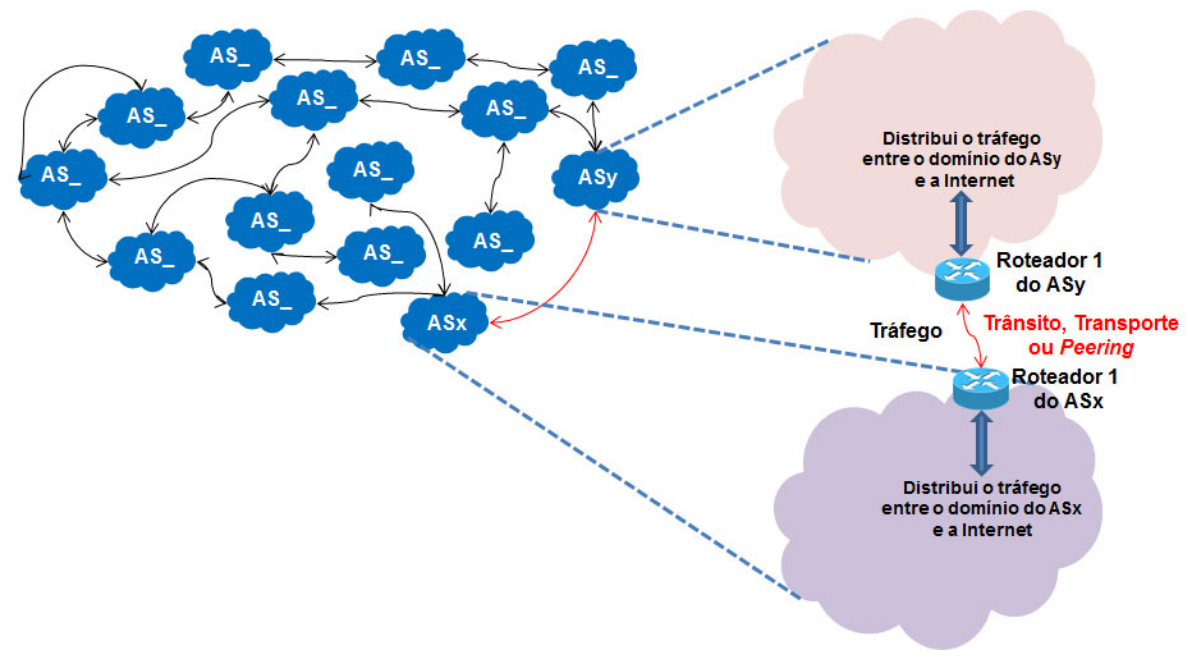

Figura 2. Interconexão entre dois ASes

Esta figura mostra o domínio de cada um dos ASes e a presença de um equipamento que permite a interconexão: o roteador. O roteador é o dispositivo no qual está instalado um protocolo apropriado para efetivar a interconexão e, compatíveis funcionalmente, entre si. A outra condição mandatória é a de que haja uma conexão física entre os dois roteadores. Conexão significa que ambos conseguem falar entre si, através de um meio de transmissão de dados. Esta transmissão ou troca de dados entre os roteadores se denomina trafego. Existem três tipos de tráfego, usualmente denominados tráfego de trânsito, tráfego de transporte e tráfego de peering. Esta classificação do tráfego tem motivação, principalmente, econômica e cada um deles é usualmente definida da seguinte maneira:

- Trânsito - é o trafego trocado entre ASes tendo como objetivo o acesso à Internet. É a mais cara das formas de tráfego. 
- Transporte - é o tráfego trocado entre ASes onde o acesso à Internet não está incluído. É uma forma de tráfego bem mais barato do que o trânsito.

- Peering - é o tráfego trocado entre ASes, onde a Internet não está diretamente envolvida e, geralmente sem nenhum valor financeiro associado. É, principalmente, um tráfego trocado por ASes, em um ambiente chamado Internet eXange(IX).

Ampliando o domínio do ASx, como mostrado na Figura 3 é possível entender que as interconexões de Sistemas Autônomos podem se estender continuamente, sem limites aparentes e confirmam que a Internet é pródiga em relação as possibilidades topológicas. Geralmente isto ocorre devido a facilidades de conexões onde fatores econômicos sejam relevantes no contexto da minimização de custos e interesses comerciais diversificados.

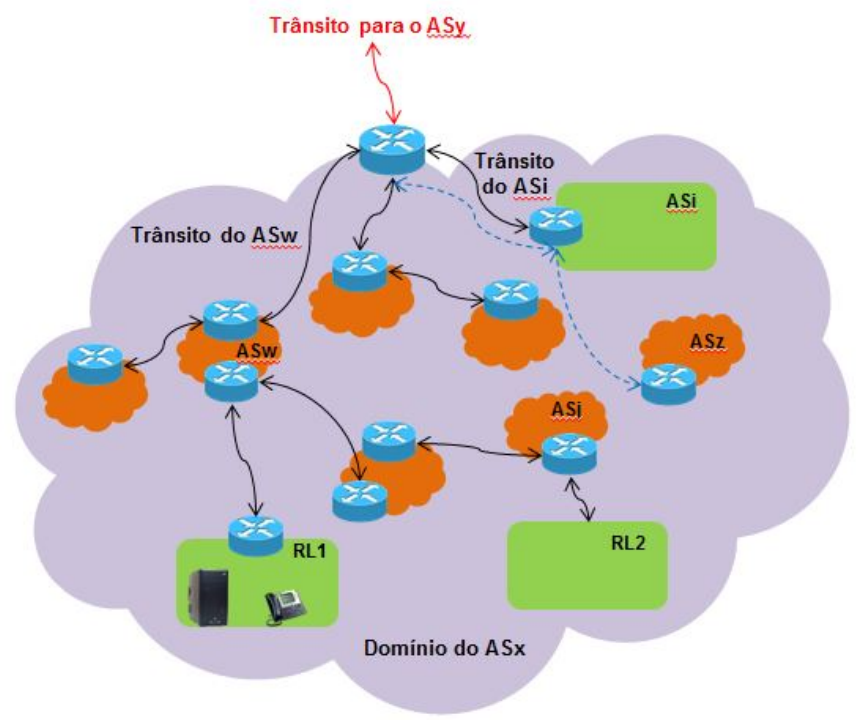

Figura 3. Rede interna (domínio) de um AS

Pela Figura 3, outros ASes podem se aproveitar das facilidades de tráfego criadas pela interconexão do ASx ao ASy, além de redes ou domínios em ambientes sem a presença de ASes, com interconexões diretas, para conviverem com os recursos da Internet.

\subsubsection{Políticas de roteamento}

Complementando a análise da definição de [Hawkinson and Bates 1996], resta identificar o significado de política de roteamento. A Figura 4 ilustra a interconexão, novamente, de dois ASes, o ASx e o ASy, em representação mais livre. Após a obtenção dos ASx e ASy via uma instituição autorizada, a cada um foi associado prefixos (ou blocos) IPv4 e/ou IPv6. Na oportunidade da interconexão, o ASx informou ao ASy que sua política de roteamento era anunciar o seu(s) respectivo(s) prefixo(s) e solicitou que ele a aceitasse.

O mesmo fez ASy, em relação ao ASx. Tais políticas de roteamento, assim definidas permitem que a Rede 1 (domínio do ASx) reconheça a Rede 2 (domínio do ASy) e vice-versa. 


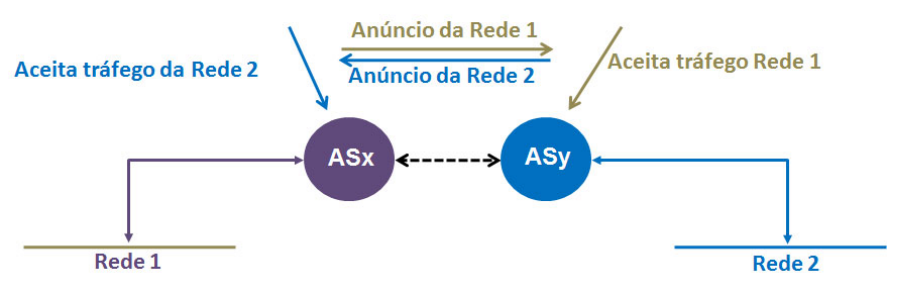

Figura 4. Política de roteamento

Grande parte das políticas de roteamento são recomendações definidas em documentos do IETF denominados Request For Comments (RFCs) e historicamente caracterizadas pelas denominadas Best Current Practices (BCPs) [Postel et al. 1995]. As políticas envolvem técnicas mais apuradas, sobretudo com o foco em segurança e exigem cuidadosos cenários que se recomenda ao administrador de um AS respeitar. Por exemplo, alguns prefixos devem ser impedidos (bloqueados) de penetrar nas respectivas redes ou algumas ações particulares devem ser impostas baseadas em características específicas do domínio. Políticas de roteamento são alvos de técnicas colecionadas sobre o nome de engenharia de tráfego ${ }^{2}$, que cuidam dos aspectos de controle, de eficiência, de otimização, de monitoramento e de segurança da Internet.

\subsubsection{Governança da Internet}

A Internet é uma organização sem controle central. Entretanto, para que ela funcione adequadamente, algumas recomendações e padrões foram e devem continuar sendo desenvolvidos. Isto é feito por diversas organizações, que em conjunto constituem o mecanismo de governança da Internet. Tais organizações são muitas e formam um complexo sistema que oferece as facilidades e recursos dirigidos aos administradores de ASes, ou como chamados neste trabalho, administradores da Infraestrutura da Internet.

As funções e atividades de cada uma destas representações afetam diretamente os administradores da Infraestrutura da Internet e indiretamente, os seus usuários, com reflexos no funcionamento da Internet, como um todo. Na Figura 5, uma representação parcial do ecossistema da Infraestrutura da Internet, o IETF [Braga et al. 2014] é um dos responsáveis por recomendar o uso de números, nomes e outros identificadores de recursos associados à Internet, como por exemplo, os protocolos. Tais recursos são encaminhados para o Internet Assigned Numbers Authority ${ }^{3}$ (IANA). O IANA, por sua vez delega esses recursos aos Regional Internet Registers (RIRs).

Os RIRs, de forma organizada distribuem tais recursos aos National Internet Registers (NIRs) ou aos Local Internet Registers (LIRs). Os LIRs são os Sistemas Autônomos, responsáveis pela formação da Internet. Toda esta estrutura existe com o objetivo de garantir que os números, nomes e identificadores usados na Internet sejam únicos.

\footnotetext{
${ }^{2}$ Awduche, D., Chiu, A., Elwalid, A., and Widja, I. (2014). Overview and principles of internet traffic engineering. Disponível em https: / / tools. ietf.org/rfc/rfc3272.txt. Acesso em 18/10/2014

${ }^{3}$ Disponível em http: / / www. iana org/numbers. Acesso em 30/05/2015
} 


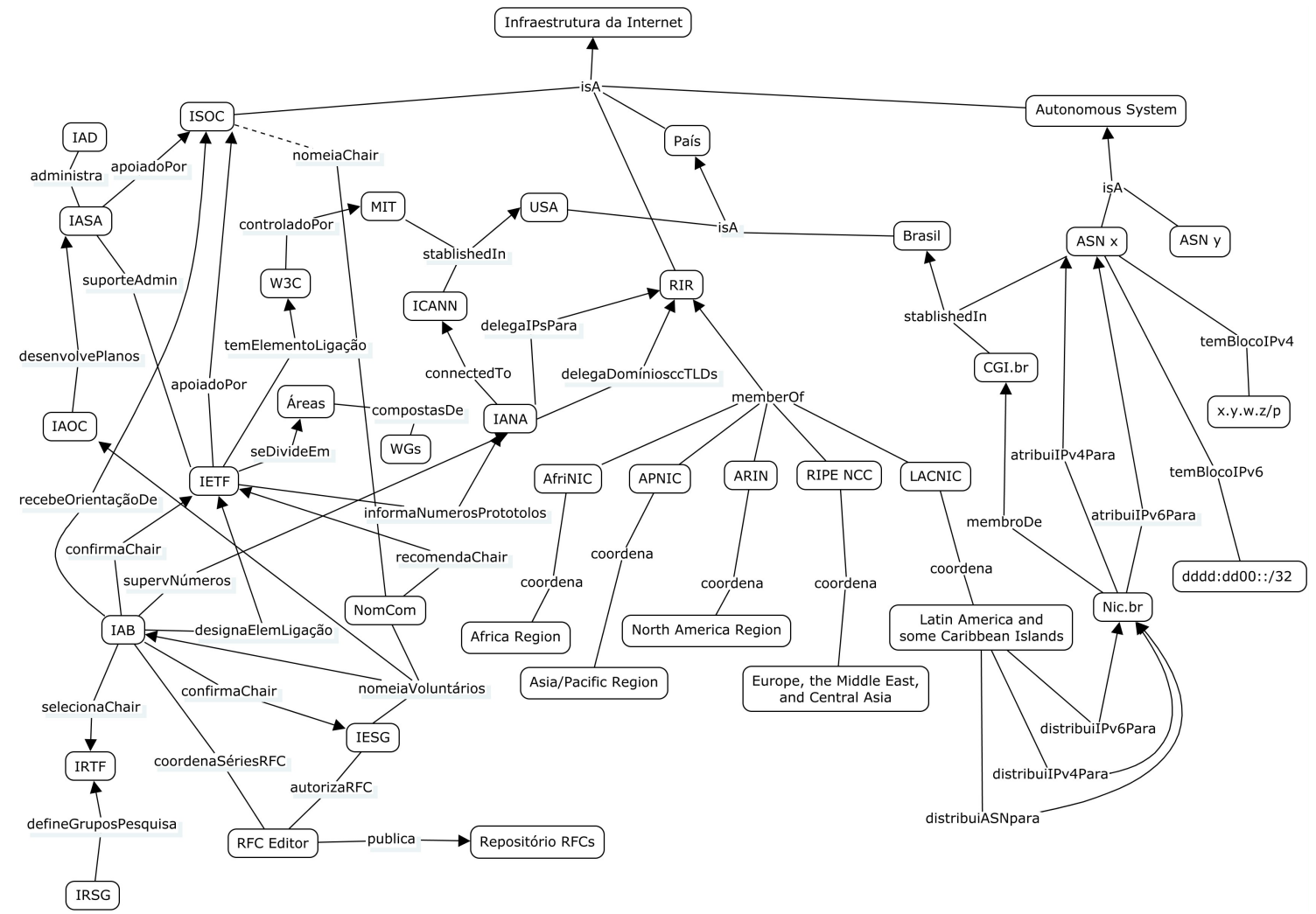

Figura 5. Visão parcial do ecossistema da Infraestrutura da Internet. Fonte: [Braga and Omar 2014]

\section{Referencial teórico}

\subsection{Elementos Inteligentes}

Elementos Inteligentes são agentes inteligentes. Segundo [Russel and Norvig 2010], "um agente (inteligente) é alguma coisa que tem percepção de seu ambiente através de sensores e age sobre o ambiente através de atuatores".

No contexto deste trabalho, os elementos inteligentes podem ser classificados de acordo com a tabela exibida na Figura 6.

O Elemento Inteligente Autônomo, que [Agoulmine 2010] diz ser a liberdade definitiva carrega a propriedade de auto-organização, cujo comportamento pode ser resumido conforme a representação da Figura 7.

Se a estabilidade do elemento inteligente autônomo é afetada por alguma ação externa (do ambiente), uma reação é imediata, que por sua própria iniciativa estabelece um comportamento apropriado, criando as condições para retornar à estabilidade original. Tal reação de um IE é baseada no auto-conhecimento e, particularmente, na capacidade de auto-aprendizagem.

Se, entretanto, para exercer a função de auto-organização, o elemento inteligente depender de uma orientação humana, não diretamente, mas através de parâmetros prédefinidos indicando como reagir, então ele passa a ser denominado Elemento Inteligente Autonômico. Dito de uma forma diferente, um elemento inteligente é autonômico se há orientação humana (um plano de alto nível), indicando como se organizar, ao reagir a 


\begin{tabular}{|c|c|c|c|c|}
\hline Tipos & Propriedades auto-* & Atividade & $\begin{array}{l}\text { Intervenção } \\
\text { Humana }\end{array}$ & Exemplos \\
\hline Autônomo & Auto-organização & $\begin{array}{l}\text { Proativo } \\
\text { Distribuído }\end{array}$ & Nenhuma & $\begin{array}{l}\text { Autonomous Nervous } \\
\text { System [1] } \\
\text { Sondas Espaciais [2] }\end{array}$ \\
\hline Autonômico & $\begin{array}{l}\text { Auto-gestão e propriedades auto-* } \\
\text { associadas }\end{array}$ & $\begin{array}{l}\text { Reativo } \\
\text { Distribuído }\end{array}$ & $\begin{array}{l}\text { Baseadas em } \\
\text { políticas e planos }\end{array}$ & $\begin{array}{l}\text { OSPF, IS-IS [11] } \\
\text { DNS }\end{array}$ \\
\hline Automático & Algumas propriedades auto-* & $\begin{array}{l}\text { Reativo } \\
\text { Distribuído }\end{array}$ & $\begin{array}{l}\text { Totalmente } \\
\text { desenvolvido por } \\
\text { humanos }\end{array}$ & BGP, DNS, IRR \\
\hline Legado & Praticamente nenhuma & $\begin{array}{l}\text { Disponível no } \\
\text { domínio } \\
\text { (ambiente) }\end{array}$ & $\begin{array}{l}\text { Dependente da } \\
\text { intervenção } \\
\text { humana, antes, } \\
\text { durante e depois }\end{array}$ & $\begin{array}{l}\text { Hosts.txt } \\
\text { IRR }\end{array}$ \\
\hline
\end{tabular}

Figura 6. Tipos de Elementos Inteligentes. Adaptada de: [Horn 2001], [Insaurralde and Vassev 2015], [Bezerra and Martin 2014], [Samaan and Karmouch 2009], [Agoulmine 2010], [Ebeling and Feistel 2011], [Behringer et al. 2015] e [Schmid et al. 2006]

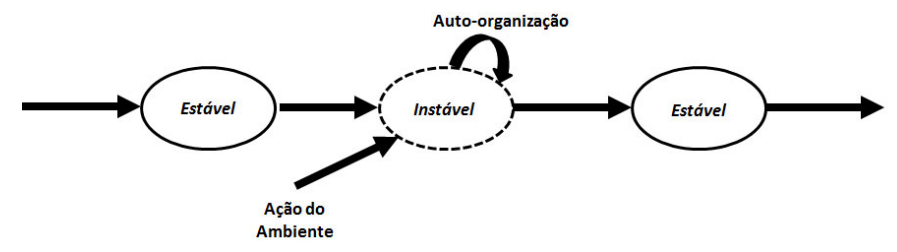

Figura 7. Auto-organização

uma ação do ambiente. Se, um elemento inteligente, para exercer sua capacidade de se organizar executa funções integralmente postas pelo ser humano, então ele é considerado um Elemento Inteligente Automático. Finalmente, se o elemento inteligente possuir ou não, as condições para se organizar, isto é, sob uma ação incomum do ambiente ele eventualmente falha, então se diz que é um Elemento Inteligente Legado.

Outra maneira complementar a classificação acima é a indicação de graus de independência e inteligência agregados aos elementos inteligentes, conforme ilustra a Figura 8 .

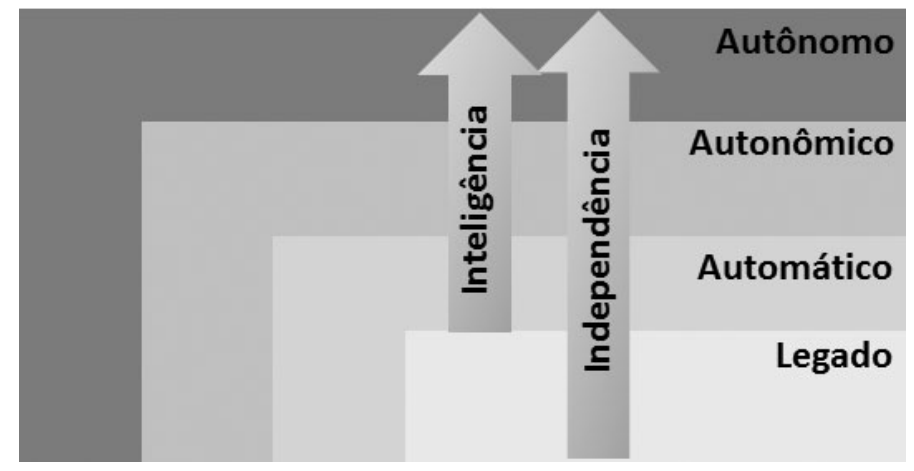

Figura 8. Caracterização de inteligência e independência dos Elementos Inteligentes

A noção de independência está vinculada à participação humana. Quanto maior a necessidade de tal participação, menos autônomo o sistema é. A noção de inteligência está associada à capacidade de auto-organização do sistema, ou seja, quanto maior a capacidade de usar eficazmente as propriedades e funcionalidades da auto-organização, mais 
autônomo o sistema é. Nem sempre haverá interesse pelos aspectos funcionais dos elementos inteligentes legados, mas será conveniente compartilhar resultados, parâmetros de configuração e, até mesmo, funcionalidades desses sistemas, com o objetivo de manter equilíbrio entre todos os elementos inteligentes envolvidos, na busca dos respectivos objetivos.

\subsection{Domínios}

Domínio representa uma coleção de coisas (atores, entidades, etc.) que estão alinhados e unidos em torno de objetivos comuns, nos limites específicos de uma determinada área de interesse $e^{4}$. No contexto deste artigo, o domínio é o ambiente delineado pelas atividades associadas ao AS.

Os ASes, por outro lado possuem subconjuntos de interesses que são identificados como subdomínios.

\subsection{Interoperabilidade e Ontologia}

Interoperabilidade é o termo usado para designar a capacidade de cooperação entre domínios e/ou subdomínios através dos respectivos elementos inteligentes, na realização de objetivos comuns. Existem dois tipos de interoperabilidades: interoperabilidade sintática e interoperabilidade semântica.

A interoperabilidade sintática se refere às informações que são trocadas entre os IEs durante a conexão. Tais informações, de um modo geral estão inseridas no contexto dos protocolos que permitem tal conectividade. Eventualmente, informações adicionais, que ascendem às camadas superiores do modelo TCP/IP usam informações adicionais para manter a conexão que não precisam de interpretação de significado, ou seja, são apenas dados no estado puro. Neste caso, alguns recursos como, por exemplo, a eXtensible

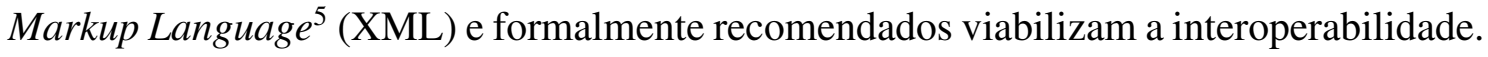

Os IEs, após a interconexão precisam manter o processo de comunicação e cooperação entre eles. Para isto serve a interoperabilidade semântica. A troca de informações entre eles passa a produzir dados com significado compreensível, cuja interpretação é apropriada para produzir os resultados esperados, durante todo o processo. Tais dados, em repositórios adequados, estão acompanhados de uma formatação especial a que se denomina de ontologia. Ontologia é o recurso utilizado para representar conhecimento interpretável por máquinas e / ou por programas. A ontologia, isto é, o conhecimento associado a dados em estado puro possui representações (linguagens) adequadas para atender a seus propósitos. Tais representações variam em função da sua capacidade de expressar claramente, o conhecimento desejado. As principais representações são recomendações padrões do $\mathrm{W} 3 \mathrm{C}^{6}$, e fazem parte da chamada Web Semântica [Breitman 2010]. Esses padrões da W3C, no conjunto fornecem facilidades e condições para construir modelos comuns de dados, compartilhar tais modelos através de metadados, caracterizar a reutilização de dados e unificar bases de dados.

Os elementos inteligentes usarão esses recursos para a transferência, troca e aquisição de conhecimento.

\footnotetext{
${ }^{4}$ http://www.ncoic.org/cross-domain-interoperability

5 http://www.w3.org/XML/

${ }^{6}$ http://www.w3.org/e http://www.w3.org/2001/sw/
} 


\subsection{Outras áreas de conhecimento envolvidas}

Autonomia envolve diversas áreas do conhecimento, tais como, entre outras:

- Engenharia do conhecimento, da qual faz parte a Web Semântica e, inclui as técnicas de aprendizagem de máquina;

- Redes Neurais e técnicas adjacentes em mineração de dados;

- Autômato Celular;

- Computação Natural;

- Modelagem de sistemas de computação.

Envolvimento interdisciplinar implica na disposição de cooperação intensa no ambiente de pesquisa e desenvolvimento. Dai a necessidade de mecanismos de divulgação e compartilhamento dos avanços alcançados sobre o modelo proposto.

\section{Trabalhos relacionados}

A Tabela 1 apresenta os principais trabalhos, que incluem modelos que foram considerados durante o desenvolvimento do modelo ANARD.

\begin{tabular}{|c|c|c|c|c|}
\hline Características & MAPE-k & Diversos & Schmid & ANIMA \\
\hline Referência & IBM [Horn 2001] & [Movahedi et al. 2012] & [Schmid et al. 2006] & $\begin{array}{l}\text { [Behringer et al. 2015] (IRTF }+ \\
\text { IETF) }\end{array}$ \\
\hline Domínio & $\begin{array}{l}\text { Da aplicação. } \\
\text { Produtos IBM }\end{array}$ & $\begin{array}{l}\text { Da aplicação. Arquitetura } \\
\text { Autonômica }\end{array}$ & Redes & $\begin{array}{l}\text { Redes: nós autonômicos com } \\
\text { mesma intenção }\end{array}$ \\
\hline $\begin{array}{l}\text { Integração entre } \\
\text { elementos }\end{array}$ & $\begin{array}{l}\text { Através de um } \\
\text { executor }\end{array}$ & $\begin{array}{l}\text { Acompanham o MAPE-K: } \\
\text { equivalente executor }\end{array}$ & Variação do MAPE-K & $\begin{array}{l}\text { Se necessário, usa o Ciclo de } \\
\text { Retroalimentação }\end{array}$ \\
\hline ID & Indefinido & Indefinido & Indefinido & IPv6 (interface do hospedeiro \\
\hline $\begin{array}{l}\text { Interferência } \mathrm{Hu}- \\
\text { mana }\end{array}$ & $\begin{array}{l}\text { Objetivo de Alto } \\
\text { Nível }\end{array}$ & Objetivo de Alto Nível & $\begin{array}{ll}\text { Objetivo de Alto Nível. } \\
\text { Comportamento } & \text { Deter- } \\
\text { minístico } & \\
\end{array}$ & $\begin{array}{l}\text { Intenção. Plano de Controle Au- } \\
\text { tonômico }\end{array}$ \\
\hline Específicas & Autogestão & Autogestão & $\begin{array}{lll}\begin{array}{l}\text { Autogestão } \\
\text { adaptação }\end{array} & \text { e } & \text { auto } \\
\end{array}$ & $\begin{array}{l}\text { Autogestão, conhecimento da } \\
\text { rede autoconhecimento (auto- } \\
\text { consciência) }\end{array}$ \\
\hline Arquitetura & $\begin{array}{l}\text { Centralizada, Es- } \\
\text { calabilidade res- } \\
\text { trita }\end{array}$ & $\begin{array}{l}\text { Hierárquica, peer, escala- } \\
\text { bilidade restrita }\end{array}$ & $\begin{array}{l}\text { Semelhante à proposta da } \\
\text { FIPA: Agência }\end{array}$ & Escalável no domínio \\
\hline
\end{tabular}

Tabela 1. Trabalhos relacionados

A origem das ideias associadas, inicialmente a Computação Autonômica surgiu da proposta elaborada por Paul Horn [Horn 2001]. Os autores de [Movahedi et al. 2012] exibem detalhes do modelo MAPE-K de Horn com seus respectivos ciclos de controle e comparam diversas outras propostas de arquiteturas autonômicas com ênfase em redes. [Schmid et al. 2006] propõe alterações no modelo MAPE-K, simplificando-o, para elementos de redes autonômicas. Os autores de [Behringer et al. 2015] iniciaram no grupo NMRG do Internet Research Task Force ${ }^{7}$ (IRTF), propostas que continuaram no grupo ANIMA do IETF, com estudos que estão em plena atividade, com propostas específicas para protocolos e representam os mais recentes e atualizados estudos, ativos sobre redes autonômicas. Dos modelos expostos na Tabela 1, somente o ANIMA propõe uma identificação (ID) para suas funções autonômicas, associando-as a um endereço IPv6, o que indica serem as funções autonômicas agregadas à interface do hospedeiro.

\footnotetext{
${ }^{7}$ http: //www.irtf.org
} 


\section{A arquitetura ANARD}

A arquitetura ANARD é apresentada na Figura 9 e dividida em quatro camadas, descritas a seguir. Ele atende ao interesse de estabelecer uma arquitetura de elementos inteligentes sob o domínio administrativo dos ASes, que como se sabe é a designação dada para as redes que formam a Internet.

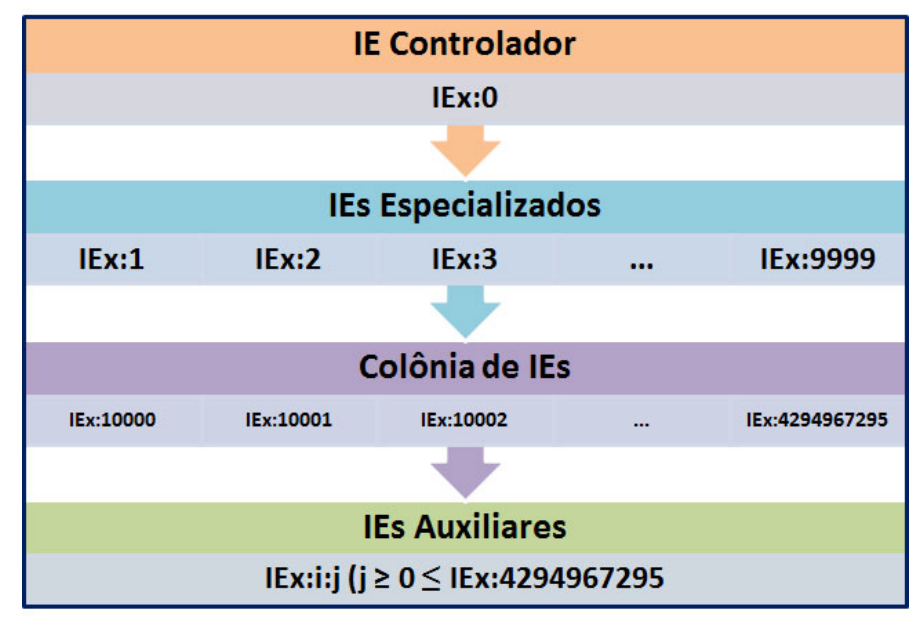

Figura 9. Modelo Abstrato do ANARD

A solução pode existir em qualquer um dos $2^{32}$ ASes possíveis [Hawkinson and Bates 1996]. Em 05/04/2015 existiam 50.126 ASes ativos na Internet (originando tráfego), de acordo com o CIDR-Report ${ }^{8}$. O número de um AS é único, controlado pelos RIRs e/ou NIRs e denomina-se Autonomous System Number (ASN). Assim, o maior valor possível de x é 50125, correspondente ao AS50125, na data acima. Não há conflito entre o modelo ser implementado em qualquer ambiente de AS e ser de domínio restrito. Na realidade, as implementações são independentes, mas com alto grau de interoperabilidade e, naturalmente, em intensa cooperação, pois os administradores de ASes dependem do comportamento de todos os outros. A IANA reservou duas faixas contíguas de números de ASes para uso privativo [Mitchell 2013]: 64512-65534 e 4200000000-4294967294. Convenientemente, estes números de ASes podem ser usados para designar Elementos Inteligentes em aplicações que necessitam de representar subdomínios.

A primeira das quatro camadas hospeda o Elemento Inteligente chamado de Controlador. Sua identificação é única e fixa: x:0, isto é, o número 0 posto ao lado direito do símbolo :, seguindo o ASN que hospeda modelo. Algumas vezes, para deixar claro qual o IE está sendo referenciado, usa-se IE antes da identificação, como por exemplo, ao afirmar que o IE Controlador é o IEx:0. Assim, se o AS5 é o domínio hospedeiro do modelo, então, o elemento controlador é o IE5:0. Nenhum IE das camadas inferiores pode existir, sem a prévia anuência do IE Controlador. Ele possui a propriedade de manter a si próprio organizado (auto-organização) e de garantir a auto-organização de qualquer IE, também, das camadas inferiores.

A segunda camada é representada pelos chamados IEs Especializados. Estes elementos são identificados por sufixos que podem ir de 1 até 9999. Os elementos especiali-

${ }^{8}$ http://www.cidr-report.org/as2.0/ 
zados servem de suporte ao IE Controlador, em atividades específicas e necessárias às respectivas funcionalidades. Tais atividades vão desde garantir a interoperabilidade de todo o sistema de IEs implementados, até o estabelecimento de funcionalidades específicas, como servidores com características fim-a-fim ${ }^{9}$, funcionalidades de acesso a banco de dados e a repositórios semânticos, software proprietários (a semelhança das APIs sulistas do SDN), recursos necessários aos IEs das camadas inferiores e muitas outras. Entretanto, o suporte ao IE Controlador é o objetivo primordial dos IEs Especializados. Este objetivo é quem determina as funcionalidades da segunda camada. Admite-se que alguns IEs Especializados possam ser Elementos Autonômicos ou elementos inteligentes que executem processos automáticos, como por exemplo, software proprietário e procedimentos associados a sistemas legados, entre outros. Um IE Especializado pode ser criado com funções que interessam somente ao IE Controlador, principalmente quando este depende das funcionalidades de IEs da terceira camada.

$\mathrm{Na}$ terceira camada reside a maior aglomeração IEs, razão pela qual é chamada de Colônia de IEs. Os elementos desta camada podem ser Autônomos, Autonômicos ou Automáticos, exceto Legados e são responsáveis diretamente pelas atividades mais importantes da aplicação, incluindo o reuso de software. Agem sob a influência de um elevado grau de interoperabilidade e cooperação entre eles e entre IEs de outras camadas e de outros domínios/subdomínios. Eles não participam diretamente de interconexões ou de troca de mensagens com outros IEs fora do domínio, mas provocam-nas, através dos IEs das camadas superiores. Há intensa atividade de interoperabilidade semântica por parte desses IEs, que possuem elevada capacidade de auto-aprendizagem devido a interações contínuas com o ambiente do domínio, e produzem efeitos de aperfeiçoamento sobre o conhecimento de outros IEs da própria colônia e dos IEs das camadas superiores, sobretudo do IE Controlador. Em outras palavras, estes IEs favorecem a aprendizagem de todo o aglomerado de IEs do modelo de camadas, que hora está sendo descrito. Os IEs, das colônias recebem uma identificação com sufixos numéricos, que vão desde o 10000 até 4294967295.

Na quarta camada estão os IEs Auxiliares. Esta camada existe, com o objetivo de permitir a transferência de demandas de computação para um novo conjunto de IEs (sucessividade do modelo). Ela reproduz, de forma sucessiva, as primeira, segunda, terceira e uma nova quarta camadas. Esta nova sequência de IEs possui um sufixo adicional :j:0 para um novo IE Controlador responsável por novas quatro camadas seguintes. Nas novas segunda, terceira e quarta camadas, as identificações dos IEs são pós fixadas com :j:id onde, j é o número do IE da Colônia que originou a nova quarta camada e o id é um número com as especificações acima. Uma típica aplicação para a quarta camada são os subdomínios, como por exemplo, as redes caseiras (homenet).

Conforme a Figura 10, os IEs estão dispostos e distribuídos entre camadas, a semelhança do que foi dito na seção anterior e são implementados no domínio de um ASN x, qualquer.

Observa-se, ainda, pela mesma Figura 10, que os IEs funcionalmente importantes nas operações inter-domínios residem nas camadas superiores.

\footnotetext{
${ }^{9}$ Reconhecido como argumentos fim-a-fim (end-to-end argumens) que estimulam o entendimento entre duas arquiteturas: o modelo de camadas e o modelo topológico [Saltzer et al. 1984]
} 


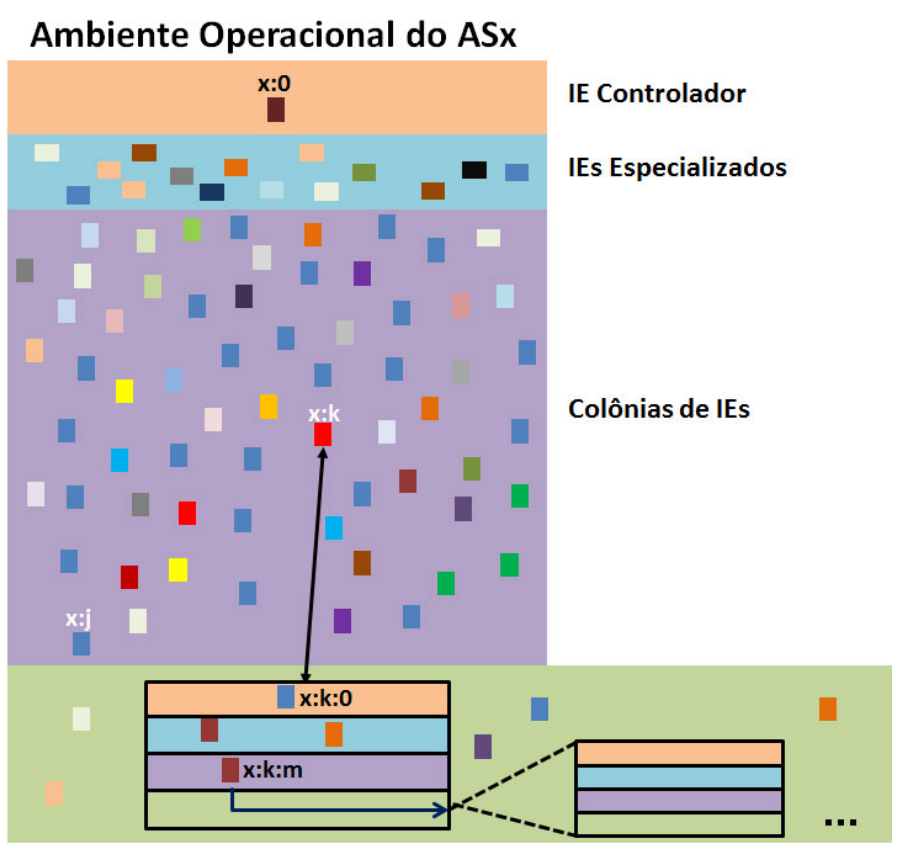

Figura 10. Modelo de implementação do ANARD

Por exemplo, uma classificação de relevância é a intensidade de agregação que um IE possui, em relação às propriedades auto-* (ou self-*). Se um IE, entretanto, possui alguma capacidade de auto-organização, ele deve participar diretamente vinculado ao IE Controlador. Mesmo participando da camada dos IEs Auxiliares, pode existir um novo IE Controlador que constrói, logicamente, uma nova arquitetura de camadas. E assim, sucessivamente.

Por outro lado, a representação do modelo é lógica (abstração da implementação física). Fisicamente, a localização de um IE no ambiente do domínio é essencial. A melhor alternativa é um endereçamento IP, preferencialmente IPv6, por razões de disponibilidade. O IE Controlador deve manter uma tabela associando a referência lógica do modelo de referência com o IP designado pelo próprio IE Controlador, a partir da premissa de que um bloco de IPv6 deve ficar disponível no início da implementação. Contudo, isto não é uma questão fundamental, pois como se verá em conclusões, em nome da segurança uma relação do IP como o ID do IE ficará disponível em um Domain Name System (DNS) privativo, o arquivo hosts alocado internamente e com ligação direta ao IE Controlador.

\section{Conclusões}

As características dos trabalhos relacionados explicitados na Tabela 1 são reproduzidas na Tabela 2, para o modelo ANARD.

O domínio restrito torna o ANARD um modelo menos complexo e mais simples ao se verificar o comportamento e permite maior confiabilidade por admitir a replicação de IEs em outros domínios. Por outro lado, um aspecto não acentua-do na Tabela 2 é a possibilidade de implementações em atividades não diretamente vinculadas a protocolos. Isto permite atender as aplicações que tendem a substituir administradores especializados e, consequentemente, eliminar trabalho adicional, custos e falhas humanas eventuais. 


\begin{tabular}{|l|l|}
\hline Características & ANARD \\
\hline Referência & Projeto atual \\
\hline Domínio & Sistemas Autônomos (ASes) \\
\hline Integração entre elementos & Ex:0, sem controle central \\
\hline ID & ID do IE \\
\hline Interferência humana & Livre para os IEs Figura 8 \\
\hline Específicas & Auto-organização, autogestão e auto adaptação \\
\hline Arquitetura & Distribuída, escalável no domínio e subdomínios \\
\hline \hline
\end{tabular}

Tabela 2. O modelo ANARD e os estudos relacionados na Tabela 1

A identificação explícita do elemento inteligente e a presença do IE Controlador estabelecem um esquema de segurança adequado e satisfatório, uma vez que somente o IP do IE Controlador é visível externamente e pode ser a interface de seu hospedeiro.

O acesso e/ou interconexão ao IE Controlador, quando por demanda de outros domínios deve possuir um mecanismo de segurança como a Resource Public Key Infrastructure (RPKI), com servidores de certificação disponíveis nos diversos RIRs, como o caso do LACNIC, que cobre, particular-mente, a região da América Latina.

Se o IE Controlador é o único IE visível, os outros IEs, embora se saiba que existem, não são localizáveis. Esta característica força o modelo a se aparentar como a Figura 11. O IE x:0, bem como qualquer IE pode replicar a si mesmo, no ambiente escuro da figura. A qualquer momento, o próprio IE poderá substituir a si próprio ou ser substituído por algum outro IE, sempre que necessário, como por exemplo, se é usado um Cyclic Redundancy Check (CRC), sobre o código do IE, na eventualidade dele sofrer alguma intervenção indevida. Este modelo de segurança recebe o nome de Segurança da Matéria Escura (SME).

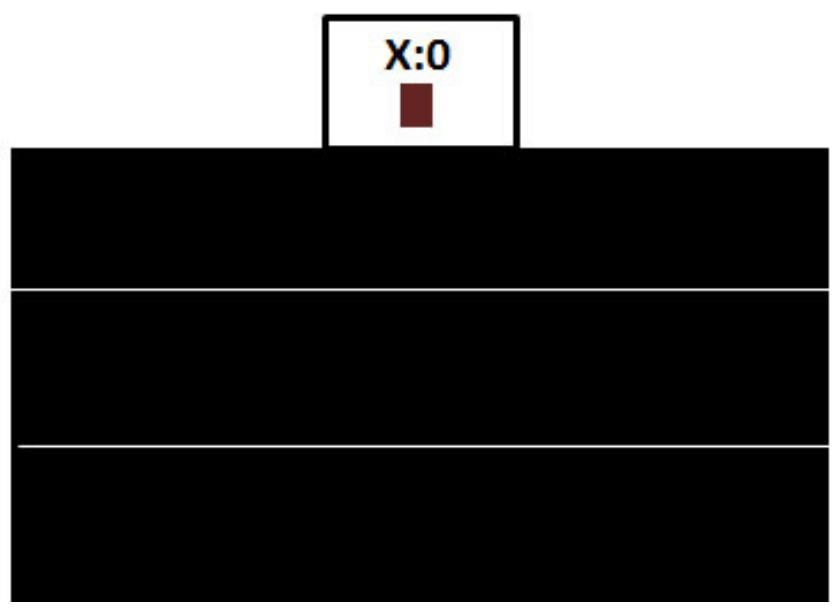

Figura 11. O IEs existem, mas não se sabe onde estão localizados

\section{Futuros desafios}

São muitos os desafios ao avanço do projeto. Entre eles, está a construção do vocabulário (conjunto de palavras e respectivos significados) que possa atender à demanda da construção de ontologias na área de Infraestrutura da Internet. O desenvolvimento deste 
vocabulário deverá ser cooperativo e, muito embora esteja havendo iniciativas de pesquisadores na área de Internet das Coisas (IoT) [Hachem et al. 2011], e pelos interessados do paradigma Internetware do projeto Context Aware Supporting Environment for Internetware ${ }^{10}$ (CASEi). Os autores consideram apropriada, uma coordenação central como o IRTF, através de um grupo de trabalho.

É recomendável o desenvolvimento de metodologia para construção de IEs, neste caso, identificados como Objetos Inteligentes (OIs). OI é um elemento inteligente construído com qualidade, reutilizável e que preserva o conhecimento de seu ciclo de vida, no contexto das aplicações do ANARD e de outros modelos relacionados. Construção de OIs, de forma adequada e padronizada induz à cooperação interdisciplinar e rapidez no desenvolvimento de IEs. Uma proposta seria usar a metodologia INTERA [Braga 2015], adaptada à construção de OIs.

Durante o desenvolvimento do estudo de caso observou-se que a noção de domínio restrito poderia ser ampliada para além dos sistemas autônomos contemplando aplicações em qualquer outro domínio. Razão pela qual recomenda-se experiências no uso do modelo proposto, em outras áreas do conhecimento, inclusive generalizações mantendo, entretanto, a restrição de domínio.

\section{Agradecimentos}

Os autores agradecem às intervenções do Prof. Dr. Ismar Frango Silveira, com contribuições relevantes ao presente trabalho.

\section{Referências}

Agoulmine, N. (2010). Introduction to autonomic concepts applied to future self-managed networks. Autonomic Network Management Principles: From Concepts to Applications, page 1.

Behringer, M., Pritikin, M., Bjarnason, S., Clemm, A., Carpenter, B., Jiang, S., and Ciavaglia, L. (2015). Autonomic networking - definitions and design goals. Technical report, IETF. IETF-ANIMA: draft-irtf-nmrg-autonomicnetwork-definitions-06.txt. Disponível em http://tools.ietf.org/pdf/ draft-irtf-nmrg-autonomic-network-definitions-06.pdf. Acessado em 15/03/2015.

Bezerra, R. M. D. and Martin, J. S. B. (2014). Network autonomic management: A tutorial with conceptual, functional and practical issues. Latin America Transactions, IEEE (Revista IEEE America Latina), 12(2):306-314.

Braga, J. (2015). Objetos de Aprendizagem: Metodologia de Desenvolvimento. Editora da UFABC, São Paulo, 1 edition.

Braga, J., Granville, L. Z., O'Flaherty, C., and Moreiras, A. M. (2014). O Livro do IETF. CGI.br, São Paulo, 1 edition.

Braga, J. and Omar, N. (2014). Semantic repository in internet infrastructure knowledge domain: Methodology. In Anais do CSBC 2014, pages 2179-2184, Brasília, DF.

\footnotetext{
${ }^{10}$ https://code.google.com/p/casei/
} 
Breitman, K. K. (2010). Web Semântica. LTC, Rio de Janeiro, 1 edition.

Ebeling, W. and Feistel, R. (2011). Physics of Self-organization and Evolution. John Wiley \& Sons.

Hachem, S., Teixeira, T., and Issarny, V. (2011). Ontologies for the internet of things. In Proceedings of the 8th Middleware Doctoral Symposium, page 3. ACM.

Hawkinson, J. and Bates, T. (1996). Report on MD5 Performance . Technical report, RFC Editor.

Horn, P. (2001). Autonomic Computing: IBM's Perspective on the State of Information Technology.

Insaurralde, C. C. and Vassev, E. (2015). Autonomic computing software for autonomous space vehicles. In Nature of Computation and Communication, pages 33-41. Springer.

Mitchell, J. (2013). Autonomous System (AS) Reservation for Private Use. Technical report, RFC Editor.

Movahedi, Z., Ayari, M., Langar, R., and Pujolle, G. (2012). A survey of autonomic network architectures and evaluation criteria. Communications Surveys \& Tutorials, IEEE, 14(2):464-490.

Nadeau, T. D. and Gray, K. (2013). SDN: Software Defined Networks. O'Reilly, USA, 1 edition.

Postel, J., Li, T., and Rekhter, Y. (1995). Best Current Practices. Technical report, RFC Editor.

Russel, S. and Norvig, P. (2010). Artificial Intelligence. Prentice Hall, New York, 3 edition.

Saltzer, J. H., Reed, D. P., and Clark, D. D. (1984). End-to-end arguments in system design. ACM Transactions on Computer Systems (TOCS), 2(4):277-288.

Samaan, N. and Karmouch, A. (2009). Towards autonomic network management: an analysis of current and future research directions. Communications Surveys \& Tutorials, IEEE, 11(3):22-36.

Schmid, S., Sifalakis, M., and Hutchison, D. (2006). Towards autonomic networks. In Autonomic Networking, pages 1-11. Springer.

Shukla, V. (2014). Introduction to Software Defined Networking. Amazon, USA, 1 edition.

Sterritt, R., Parashar, M., Tianfield, H., and Unland, R. (2005). A concise introduction to autonomic computing. Advanced Engineering Informatics, 19(3):181-187.

Wickboldt, J. A., De Jesus, W. P., Isolani, P. H., Bonato Both, C., Rochol, J., and Zambenedetti Granville, L. (2015). Software-defined networking: management requirements and challenges. Communications Magazine, IEEE, 53(1):278-285. 